multiple patient environment. Two small group exercises were used that helped prepare teams before the multiple patient scenarios.

Methods.-Interprofessional teams of 5 to 6 trainees were assessed during two environmentally challenging (ie, flood evacuation and infectious disease outbreak) emergency preparedness training scenarios. To prepare teams before the multiple patient scenarios, 2 small group exercises were developed: 1) a communications exercise utilizing a large cardboard puzzle of a disaster scene (cost, \$30); and 2) a triage exercise with mock patients utilizing 60 small handheld toys (cost, \$20). Individual and performance objectives were assessed. Before training, team members completed a 24question knowledge assessment, a demographic survey, and a comfort level self-assessment. After training, trainees repeated the 24 questions, a self-assessment, and a course assessment.

Results.-Of the 708 participants who completed the course between November 2011 and August 2014, 31.9\% were medical students, $49.9 \%$ physicians, $7.2 \%$ nurses, and less than $1 \%$ each of emergency managers, mental health providers, EMS personnel, and law enforcement officials. Overall, 708 participants (100\%), 509 (71.9\%), and 243 (34.3\%) completed the pre-test, post-test, and course assessment, respectively. The average percentage of correct answers between the pre-test and post-test increased from 46.8 to 76.3. Two hundred forty-two (99.6\%) reported the course as relevant to care providers, and $241(91.5 \%)$ highly recommended the course.

Conclusions.-Our competency-based emergency preparedness training curriculum used innovative small group exercises to improve disaster team performance, and cost less than $\$ 50$. Trainees improved their knowledge, and they highly recommended the course. The tools may serve disaster organizations lacking resources to train in the field.

\author{
Lancer A. Scott, MD \\ Layne Madden, BS \\ Judy Staub, BS \\ Jason Crumpler, BS \\ Jamal Jones, BS \\ Simon Watson, MD \\ Wade Manaker, MD \\ Blake Willis, MD \\ Charleston, SC, USA
}

response with limited resources. Remote locations inhibit communication and outside support, leading to extended time to definitive care. That results in greater responsibility for first responders. A mobile application with protocol for wilderness issues may help providers with limited knowledge to manage medical emergencies until professional medical care is possible. Improvement in first responder care can also decrease emergency medical services and hospital costs, in addition to preventing long-term complications.

Objective.-The purpose of this study is to determine, by simulated scenario performance, whether the use of a smartphone application will improve management of common medical emergencies in the wilderness.

Methods.-We identified the top-rated survival and first aid applications available in the iPhone and Android digital application markets. We designed 6 emergency scenarios set in a wilderness context with performance based on standard criteria outlined in Auerbach's Wilderness Medicine textbook. Using a prospective study, we compared patient care performance between simulations that were application assisted and simulations that were not. A population of 66 simulations of 6 scenarios completed by 11 inexperienced students provided a $64 \%$ power to detect a difference of $50 \%$ in the improvement of scores when using the wilderness medicine application. Interrater reliability was also calculated on a subset of the stations.

Results.-Performance on simulations did not vary with smartphone application use, according to $t$ test $(P=.296)$.

Conclusions.-Smartphone applications theoretically provide accessible knowledge for inexperienced first responders in remote settings. Our study suggests an opening in the smartphone application market for an effective wilderness survival tool. Furthermore, lack of training in basic first aid may not be compensated for by smartphone application help. We plan to extend this study to include more participants.

\author{
Robert Katzer, MD, MBA \\ Tanya Wilcox, BA \\ Merrick Brodsky, BS, BA \\ Monica Straatman, BA \\ Nitish Nag, BA \\ Justin Yanuck, BA \\ Irvine, CA, USA
}

\section{Smartphones in the Wild: Can Technology Improve Emergency Care in Remote Settings?}

Background.-Environmental illness and injury in the wilderness can occur quickly and unexpectedly, requiring a swift 